\title{
Foreword to the Special Issue on Pattern Recognition in Remote Sensing
}

$\mathbf{T}$ HE constant increase in the amount of remotely sensed images as well as the urgent need for the extraction of useful information from such data sets have made the development of new pattern recognition techniques a popular research topic for several decades. This data volume, together with the complexity of the content acquired by a diverse set of sensors, require new interdisciplinary work involving the application of novel pattern recognition techniques to unsolved problems in remote sensing image analysis that cannot be handled by using traditional remote sensing methods. Therefore, a wide range of pattern recognition techniques have been proposed for both traditional application areas such as land cover and land use classification, road network extraction, and agricultural mapping and monitoring, as well as more recent topics such as monitoring of human settlements, management of natural resources, response planning for natural and human-induced disasters, assessment of the impact of climate change, and conservation of biodiversity.

One of the important challenges in the combined fields of pattern recognition and remote sensing is the increasing resolution of the data that has led to an expansion in the data volume and an increase in the complexity of the analysis algorithms. Higher resolution often applies to the spatial characteristics of the images where additional patterns are visible in large scenes and, therefore, more elaborate yet faster techniques need to be developed to detect and recognize them. Furthermore, a characteristic peculiar to remote sensing is that "high" resolution may mean not only "high spatial", but also "high spectral" resolution, leading to a wealth of problems related to high dimensionality of feature spaces, and "high temporal" resolution, requiring new methods for time series analysis. Researchers also need to take into account the nature of different sensors used for collecting data with different modalities such as multi-spectral and hyper-spectral data, as well as synthetic aperture radar (SAR) data, so that proper techniques that are capable of modeling the peculiar statistical properties of each type of data are used. Finally, performance evaluation of the developed supervised, semi-supervised, unsupervised, batch and active learning algorithms is also an interesting problem given the limited availability of detailed ground truth data sets.

This special issue is associated with the 6th IAPR Workshop on Pattern Recognition in Remote Sensing (PRRS 2010) [1] that was held in Istanbul, Turkey, on August 22, 2010 in conjunction with the IAPR International Conference on Pattern Recognition (ICPR 2010) with co-sponsorship by IAPR and IEEE Geoscience and Remote Sensing Society. The PRRS Workshop, that is implemented by the IAPR Technical Committee 7 on Remote Sensing, offers an opportunity for researchers to gain a better understanding of the many diverse research topics in remote

Digital Object Identifier 10.1109/JSTARS.2012.2218673 sensing that require contributions from the pattern recognition community, and has established itself as an important event for scientists involved in the combined fields of pattern recognition and remote sensing.

The special issue follows the tradition of three earlier issues [2]-[4] for promoting the state-of-the-art pattern recognition research on the analysis of remote sensing data sets. Papers were solicited from the pattern recognition, geoscience, and remote sensing communities at large through an open call for papers. The issue is composed of the following nine papers, selected among the 30 received submissions according to the standard review process of the IEEE JOURNAL OF SELECTED TOPICS IN APPLIED EARTH OBSERVATIONS AND REMOTE SENSING (JSTARS), covering different aspects of remote sensing image analysis.

1) In [5], a procedure to adapt a classifier trained on a source image to a target image with similar spectral properties based on SVM is presented. This approach is designed to boost the performance of active learning methods when applied in the context of domain adaptation. The adaptation is carried out in two steps: Step 1 -an active selection of the pixels to be labeled is performed on a set of candidates of the target image in order to select the most informative pixels, and Step 2-the weights associated with these samples are iteratively updated using different criteria, depending on their origin (source or target image). In order to validate the proposed methodology, experiments on two VHR QuickBird images and on a hyperspectral AVIRIS image are conducted and the results are compared with existing techniques that do not involve any adjustments to the target domain. It is concluded that the best performance is obtained with the new approach.

2) In [6], a comparative study on the effects of the quantity of training examples on the classification accuracy of support vector machine (SVM), relevance vector machine (RVM), and sparse multinomial logistic regression (SMLR) classifiers is presented. The experiments using both airborne and spaceborne multispectral data sets show that the RVM and SMLR are able to achieve similar classification accuracies as the SVM but require considerably fewer training examples. The results also show that the training cases that are found to be useful by SVM and RVM have distinct and potentially predictable characteristics, and suggest a potential to design classifier-specific intelligent data acquisition activities using small training sets.

3) In [7], a link-based cluster ensemble technique is used to improve cloud classification and satellite precipitation estimation. The method consists of segmentation of infrared cloud images into patches, cloud patch feature extraction, combination of multiple data partitions from different clustering methods using a pairwise similarity cluster 
ensemble, and precipitation estimation. The experiments show that using the cluster ensemble increases the performance of rainfall estimates compared to other methods.

4) In [8], a methodology to automatically extract river structures in high resolution SAR imagery is presented. This method is based on mathematical morphology and supervised image classification, using automatically selected training samples. When applied toTerraSAR-X imagery, the proposed method achieves accurate results and performs almost similar in terms of accuracy, when compared to the other techniques that require manual user interaction. Moreover, the proposed method can be easily applied to multitemporal and multipolarized image data, thus providing flexibility to extend to larger scale applications.

5) In [9], an algorithm to extract road network for SAR images based on multiscale geometric analysis (MGA) with beamlet is proposed. The main idea is to acquire pairs of connection points between road candidates according to the guidance segments which are obtained with MGA. This process allows the capture of linear features at multiple scales based on statistical and geometrical properties. Experiment results show that the proposed method has higher completeness and quality indexes than traditional methods.

6) In [10], a new approach for sea ice segmentation in SAR images is described. The approach combines an edge-preserving region-based representation with a region-level Markov random field (MRF) model. The watershed algorithm is used to partition the image into primitive regions, and the MRF is used to model the contextual dependencies among these regions. The region-based representation largely reduces the search space during optimization, improves parameter estimation of the feature model, and leads to considerable computational savings and reduced probability of false segmentation.

7) In [11], a novel method for oil spill regions segmentation from SAR imagery is presented. This approach is based on an adaptive level set evolution process based on the IR components of the imagery. This can be achieved by combining a new signed pressure function, derived from the illumination and reflectance values present in the image with homomorphic decomposition to extract the IR components in an image. The corresponding results obtained are found to be superior when compared with traditional techniques; thus leading to more accurate identification of the oil spill regions.

8) In [12], an enhanced super-resolution mapping method that combines the positive attributes of contouring and the Hopfield neural network is presented. This enhanced method is based on fitting a contour of equal class membership to a pre-final output of a standard Hopfield neural network. The application of the proposed method to both simulated and real image data sets show that this method is more accurate than the standard contouring and Hopfield neural network based methods individually, with error typically reduced by a factor of two or more.

9) In [13], a series of analyses using both simulated and real satellite image data with the goal of assessing how land- scape patch size and parameter weighting for super resolution mapping through Hopfield Neural Network (HNN) affects land cover classification is described. An integrated approach based on the fusion of classification results derived from different $\mathrm{HNN}$ parameters setting is proposed in order to enhance the performance of the methodology for areas with a mix of large and small patches and results presented over real case high and low resolution digital and multiband images (derived from Google Earth satellite images small subsets and MODIS data over a lake region in Canada). It is shown that the method's suitability varies between landscapes, being most suited to situations in which landscape patches are large.

We are pleased to offer this special issue to the scientific community, and hope that it accomplishes our goal of highlighting recent advances in a wide range of challenges within the combined fields of pattern recognition and remote sensing. We would like to take this opportunity to thank the Editor-in-Chief of JSTARS, Prof. Jocelyn Chanussot, for his constant support to this special issue, as well as all the authors and reviewers for their outstanding contributions.

\section{NiCOLAS H. YounAn, Guest Editor \\ Department of Electrical and Computer Engineering Mississippi State University \\ Mississippi State, MS 39762 USA}

\author{
SELIM AKSOY, Guest Editor \\ Department of Computer Engineering \\ Bilkent University \\ Ankara, 06800 Turkey
}

\author{
RoGer L. KING, Guest Editor \\ Center for Advanced Vehicular Systems \\ Mississippi State University \\ Mississippi State, MS 39762 USA
}

\section{REFERENCES}

[1] S. Aksoy, N. H. Younan, and W. Forstner, in Proc. IEEE 2010 IAPR Workshop on Pattern Recognition in Remote Sensing, 2010, Catalog Number: CFP1080E-ART.

[2] P. Gamba and D. Clausi, "Preface," Pattern Recognition Lett., vol. 27, no. 4, p. 217 , Mar. 2006.

[3] D. A. Clausi, S. Aksoy, and J. C. Tilton, "Foreword to the special issue on pattern recognition in remote sensing," IEEE Trans. Geosci. Remote Sens., vol. 45, no. 12, pp. 3855-3856, Dec. 2007.

[4] S. Aksoy, N. H. Younan, and L. Bruzzone, "Pattern recognition in remote sensing," Pattern Recognition Lett., vol. 31, no. 10, pp. 1069-1070, Jul. 15, 2010.

[5] G. Matasci, D. Tuia, and M. Kanevski, "SVM-based boosting of active learning strategies for efficient domain adaptation," IEEE J. Sel. Topics Appl. Earth Observ. Remote Sens., vol. 5, no. 5, pp. 1335-1343, Oct. 2012.

[6] M. Pal and G. M. Foody, "Evaluation of SVM, RVM and SMLR for accurate image classification with limited ground data," IEEE J. Sel. Topics Appl. Earth Observ. Remote Sens., vol. 5, no. 5, pp. 1344-1355, Oct. 2012.

[7] M. Mahrooghy, N. H. Younan, V. G. Anantharaj, J. Aanstoos, and S. Yarahmadian, "On the use of a cluster ensemble cloud classification technique in satellite precipitation estimation," IEEE J. Sel. Topics Appl. Earth Observ. Remote Sens., vol. 5, no. 5, pp. 1356-1363, Oct. 2012.

[8] S. Klemenjak, B. Waske, S. Valero, and J. Chanussot, "Automatic detection of rivers in high-resolution SAR data," IEEE J. Sel. Topics Appl. Earth Observ. Remote Sens., vol. 5, no. 5, pp. 1364-1372, Oct. 2012. 
[9] C. He, "Road extraction from SAR imagery based on multiscale geometric analysis of detector responses," IEEE J. Sel. Topics Appl. Earth Observ. Remote Sens., vol. 5, no. 5, pp. 1373-1382, Oct. 2012.

[10] X. Yang and D. A. Clausi, "Evaluating SAR sea ice image classification using edge-preserving region-based MRFs," IEEE J. Sel. Topics Appl. Earth Observ. Remote Sens., vol. 5, no. 5, pp. 1383-1393, Oct. 2012.

[11] G. R. Reddy, N. Baddiri, Z. Syed, and R. R. Rao, "Segmentation of oil spill images with IR based adaptive level set model," IEEE J. Sel. Topics Appl. Earth Observ. Remote Sens., vol. 5, no. 5, pp. 1394-1402, Oct. 2012.
[12] Y.-F. Su, G. M. Foody, A. M. Muad, and K.-S. Cheng, "Combining Hopfield neural network and contouring methods to enhance superresolution mapping," IEEE J. Sel. Topics Appl. Earth Observ. Remote Sens., vol. 5, no. 5, pp. 1403-1417, Oct. 2012.

[13] A. M. Muad and G. Foody, "Impact of land cover patch size on the accuracy of patch area representation in HNN-based super resolution mapping," IEEE J. Sel. Topics Appl. Earth Observ. Remote Sens., vol. 5, no. 5, pp. 1418-1427, Oct. 2012.

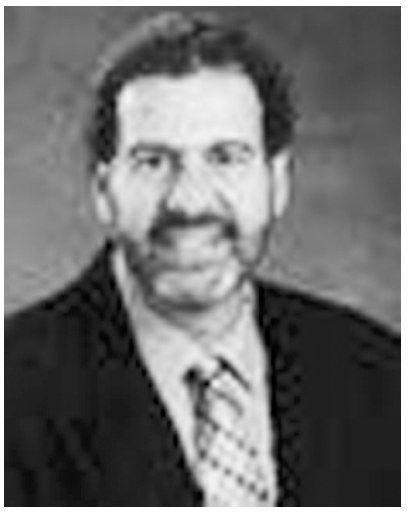

Nicolas H. Younan (S'87-M'88-SM'99) received the B.S. and M.S. degrees from Mississippi State University in 1982 and 1984, respectively, and the Ph.D. degree from Ohio University in 1988.

He is currently the Department Head and James Worth Bagley Chair of Electrical and Computer Engineering at Mississippi State University. His research interests include signal processing and pattern recognition. He has been involved in the development of advanced image processing and pattern recognition algorithms for remote sensing applications, image/data fusion, feature extraction and classification, automatic target recognition/identification, and image information mining.

Dr. Younan has published over 200 papers in refereed journals and conference proceedings, and book chapters. He has served as the General Chair and Editor for the 4th IASTED International Conference on Signal and Image Processing, Co-Editor for the 3rd International Workshop on the Analysis of Multi-Temporal Remote Sensing Images, Guest Editor, Pattern Recognition Letters, and JSTARS. He is a senior member of IEEE and a member of the IEEE Geoscience and Remote Sensing society, serving on two technical committees: Data Fusion, and Data Archive and Distribution. He also served as Co-Chair for the Workshop on Pattern Recognition for Remote Sensing (PRSS 2008 and 2010).

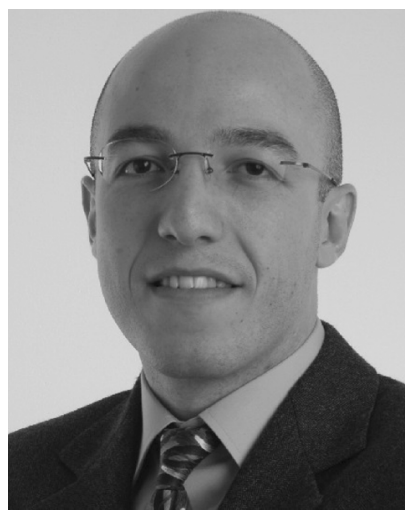

Selim Aksoy (S'96-M'01-SM'11) received the B.S. degree from the Middle East Technical University, Ankara, Turkey, in 1996 and the M.S. and Ph.D. degrees from the University of Washington, Seattle, in 1998 and 2001, respectively.

He has been an Assistant Professor at the Department of Computer Engineering, Bilkent University, Ankara, since 2004, where he is also the Co-Director of the RETINA Vision and Learning Group. Before joining Bilkent, he was a Research Scientist at Insightful Corporation, Seattle, where he was involved in image understanding and data mining research sponsored by the National Aeronautics and Space Administration, the U.S. Army, and the National Institutes of Health. During 1996-2001, he was a Research Assistant at the University of Washington, where he developed algorithms for content-based image retrieval, statistical pattern recognition, object recognition, graph-theoretic clustering, user relevance feedback, and mathematical morphology. During the summers of 1998 and 1999, he was a Visiting Researcher at the Tampere International Center for Signal Processing, Tampere, Finland, collaborating in a content-based multimedia retrieval project. His research interests include computer vision, statistical and structural pattern recognition, machine learning and data mining with applications to remote sensing, medical imaging, and multimedia data analysis.

Dr. Aksoy is a senior member of the IEEE Geoscience and Remote Sensing Society, the IEEE Computer Society, and a member of the International Association for Pattern Recognition (IAPR). He received the CAREER Award from the Scientific and Technological Research Council of Turkey (TUBITAK) in 2004. He was one of the Guest Editors of the special issues on Pattern Recognition in Remote Sensing of IEEE Transactions on Geoscience and Remote Sensing, Pattern Recognition Letters, and IEEE Journal of Selected Topics in Applied Earth ObServations and Remote Sensing in 2007, 2009, and 2012, respectively. He is an Associate Editor of Pattern Recognition Letters. He served as the Vice Chair of the IAPR Technical Committee 7 on Remote Sensing during 2004-2006, and as the Chair of the same committee during 2006-2010. 


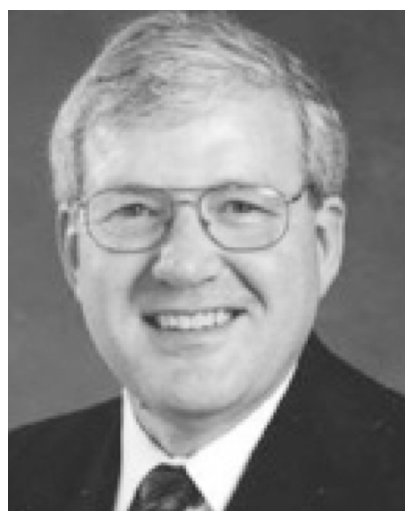

Roger L. King (S'70-M'76-SM'92) received the B.S. degree from West Virginia University, Morgantown, WV, in 1973 and the M.S. degree in electrical engineering from the University of Pittsburgh, Pittsburgh, PA, in 1978. He received the Ph.D. in engineering from the University of Wales, Cardiff, in 1988.

He began his career with Westinghouse Electric Corporation, but soon moved to the U.S. Bureau of Mines Pittsburgh Mining and Safety Research Center. Upon receiving his Ph.D. in 1988 he accepted a position in the Department of Electrical and Computer Engineering at Mississippi State University where he holds the position of Giles Distinguished Professor. At Mississippi State University, he serves as the Director of the Center for Advanced Vehicular Studies in the Bagley College of Engineering which is a member institute of the High Performance Computing Collaboratory (HPC).

Dr. King has received numerous awards for his research including the Department of Interiors Meritorious ServiceMedal. Over the last 30 years, he has served in a variety of leadership roles with the IEEE Industry Applications Society, Power and Energy Society, and Geosciences and Remote Sensing. He has served for four years as the Chair of the IEEE GRSS Data Archiving and Distribution Technical Committee and served as a member of the IEEE GRSS AdCom. He also served as the Co-Technical Chair for IGARSS 2009 in Cape Town, South Africa. He is a member of the European Image Information Mining Coordination Group (IIMCG) and Senior Member of IEEE. 